\title{
Male catheter insertion simulation using a low-fidelity 3D-printed model in undergraduate medical learners
}

\author{
Charlie Gillis, BSc', David Harvey, MD², Nicole Bishop, MSc (Kin)³, Greg Walsh, \\ B.Eng (Mechanical) ${ }^{3}$, Adam Dubrowski, Ph.D. ${ }^{4}$ \\ I. Class of 2020, Faculty of Medicine, Memorial University, St. John's, Newfoundland, Canada \\ 2. Department of Surgery (Urology), Memorial University, St. John's, Newfoundland, Canada \\ 3. Department of Biomedical Engineering, Memorial University, St. John's, Newfoundland, Canada \\ 4. Department of Emergency Medicine, Memorial University, St. John's, Newfoundland, Canada
}

\begin{abstract}
Urinary catheter insertion is one of the most widely performed procedures in a clinical setting. Inexperienced catheterizations constitute a high percentage of urethral trauma in hospital settings, with as high as $75 \%$ of comorbidities related to inaccurate insertion. Simulation training can help learners feel more confident, shorten the learning curve, and provide a safe learning environment for novices to make, and learn from, mistakes. Three dimensional (3D)printed simulation models are as effective as commercially available models for novice learners, and have the benefits of being inexpensive, anatomically correct, portable and can be easily modified and rapidly produced as needed. A 3D-printed male urinary catheter insertion simulation model, designed by MUNMed 3D, was offered to Memorial University medical students as part of pre-clerkship procedural training. Fourteen students were provided with a checklist for the procedure and the 3D-printed urinary catheter insertion simulator, and following the simulation, were asked to complete a 5-point Likert survey on their experience. The average self-reported skill before using the model was 1.29 (out of 5), which increased to 3.2I (out of 5). All 14 respondents selected either "agree" or "strongly agree" for the following four survey items: the simulation was an accurate anatomical representation, they would prefer learning on this simulation model before performing this procedure, they would recommend the model to other learners, and they found this model beneficial overall. Simulation training with a 3D-printed urinary catheter insertion simulator allows trainees the opportunity to become confident and familiarize themselves with the procedure before performing it on a real patient.
\end{abstract}

\section{Introduction}

Urinary catheter insertion is a widely-performed procedure in the hospital setting for patients who lack bladder function or require a fluid status assessment. ${ }^{1,2}$ To perform this medical procedure, a hollow tube is inserted through the urethra and into the bladder; once inside the bladder, the end of the catheter is inflated to prevent spontaneous removal. The inserted catheter allows for passage of urine out of the body, or for urine measurement when considering the intake and outflow of fluids. Although widely performed in the hospital setting by various healthcare practitioners, this procedure is expected to be performed by Canadian undergraduate medical students as they rotate through the clinical setting. ${ }^{3}$ As with any invasive procedure, there are risks and complications associated with catheter insertion. Improper catheterization technique can result in urethral trauma and may contribute to catheter-related urinary tract infections. ${ }^{3,4}$ In some centres, up to $75 \%$ of reported urethral trauma following a catheterization is caused by learners, highlighting the need for early and efficient training in this procedure. ${ }^{5}$ Traumatic catheter insertion is recognized as a major cause of iatrogenic urethral strictures, resulting in obstructive or irritative urinary symptoms that have a profound impact on patients' quality of life. ${ }^{6}$
With healthcare focusing on patient safety and successful outcomes, a need has been identified to achieve these goals in the area of the urinary catheter insertion procedure. As mentioned, urinary catheter insertion is a mandatory skill for medical students in their clinical years of undergraduate training. The students should complete the procedure under direct supervision of their preceptors to ensure accurate completion of the procedural steps in the correct order, with sterility preserved. To prepare for procedural training and the clinical clerkship experience, many medical schools provide a "pre-clerkship" training course that offers simulation for commonly performed medical procedures. Despite being one of the most prevalent procedures ${ }^{1}$, lack of experience with urinary catheter insertion is an ongoing concern for undergraduate learners at the pre-clerkship level. This learning gap can be fulfilled using three-dimensional (3D) printed simulation models, allowing students to practice the steps of the procedure and familiarize themselves with this technique. This simulation training can assist learners in feeling more confident when performing urinary catheterization, reducing the potential for complications that can occur as a result of inexperience. ${ }^{7}$ Simulation training allows trainees to make mistakes without the implications of litigiousness 
or causing harm, which offers a strong learning experience for skills development. ${ }^{8}$ Specific to urinary catheter insertion, simulation training has been shown to increase user confidence which offers a significant advantage when first facing an insertion in an evaluated clinical setting. ${ }^{7}$

Three-dimensional printing is becoming more prevalent throughout the medical fields due to technological advancements and decreased production costs. ${ }^{9}$ In simulation-based medical education (SBME), 3D printing has several distinct advantages. In healthcare centres that have access to $3 \mathrm{D}$ printing technology, a design can be printed as a point-of-care simulation without the need for expensive commercial grade models; this would be especially beneficial in rural and remote areas. Indeed, 3D printed simulators are less expensive than most simulation kits commercially available, but can be just as effective for the acquisition of skills in novice learners. ${ }^{10}$ Due to the ability to print anatomically correct models, 3D printing models are being widely incorporated into simulation training in multiple surgical fields and other specialties. ${ }^{11,12}$ This is also ideally suited for medical learners to familiarize themselves with the steps of a procedure without risk of patient adverse events.

The aim of this project was to help fill an educational gap identified in the undergraduate medical school curriculum for urinary catheter insertion. A low-cost, 3D printed simulation model was developed to assist undergraduate learners in the acquisition of confidence and procedural skill knowledge prior to clerkship rounds in a hospital setting. This article describes the development of the male urinary catheter insertion simulator, the feedback obtained from novices on the design, and concludes by presenting a framework for future developments of the 3D printed urinary catheter simulation model.

\section{Methodology}

The male urinary catheter insertion simulation model was designed under guidance of a staff urologist (Figure 1). The model was $3 \mathrm{D}$ printed using an Ultimaker 3, with $20 \%$ infill and $0.2 \mathrm{~mm}$ layer height. The base, water reservoir (urinary bladder) and mounting structures were printed using red polylactic acid (PLA) and the external genitals were cast in Smooth-On 00-30 silicone. The urethral component was printed with polyurethane thermoplastic elastomer (TPE) in durometer 80A. The overall cost of the model was approximately $\$ 35$, with the silicone external genitals costing $\$ 30$ and the rest of the print materials being less than $\$ 5$. This estimate does not include the initial design time.

The urinary catheter insertion simulation was carried out by preclerkship students during procedural training, where the model was set up in a separate unsupervised area of the simulation lab for participants to practice with on their own time. The study was described to participants, who consented to the study by completing the survey. A checklist was provided to the participants to guide the participants through the correct steps of urinary catheter insertion. The checklist used was incorporated from a validated Delphi survey and contribution from a staff urologist. ${ }^{13}$ Participants were provided with a catheter insertion kit and given up to ten minutes to practice the steps of the procedure using the model. Following the use of the model, a survey was provided to the participants to complete. Questions were asked using a 5-point Likert scale and were intended to assess how the learner's confidence and experience changed with use of the model, the anatomical accuracy of the model and their overall impression of the model. Participants were presented with a series of statements related to these objectives and were asked to select on a 5-point bipolar Likert scale their level of agreement with the statement (strongly disagree to strongly agree).

This project is a quality improvement initiative in the procedural training of urinary catheter insertion, and whether the implementation of a low-cost simulation would be beneficial to learners. As such, questions were designed to generate qualitative information about the

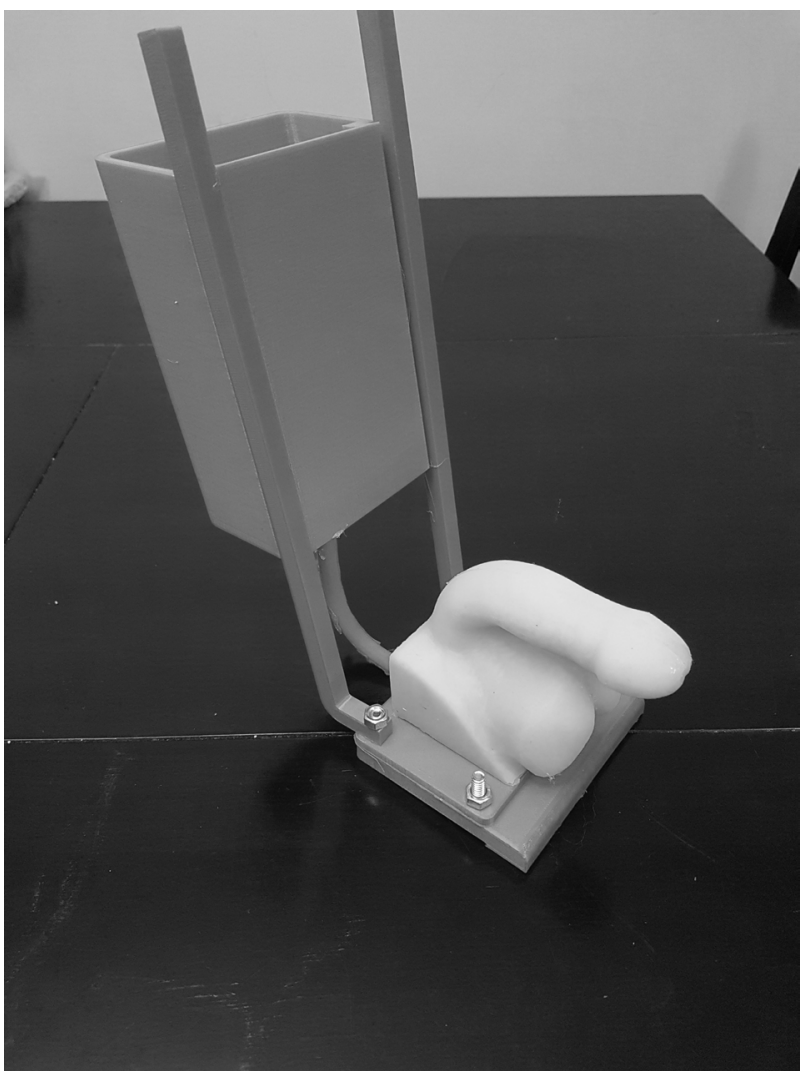

Figure I: 3D-Printed Urinary Catheter Insertion Model 
Table I: Post-simulation survey results from preclerkship trainees.

\begin{tabular}{|c|c|c|c|c|c|c|}
\hline Survey Component & $\mathbf{I}$ & 2 & 3 & 4 & 5 & Mean \\
\hline I.What was the level of your skill before using the simulation? & II & 2 & I & 0 & 0 & 1.29 \\
\hline 2. What was the level of your skill after using the simulation? & 0 & 1 & 9 & 4 & 0 & 3.21 \\
\hline 3. The catheter insertion model felt realistic. & 0 & 0 & 7 & 3 & 4 & 3.79 \\
\hline 4. This simulation was an accurate representation of the relevant anatomy. & 0 & 0 & 0 & 11 & 3 & 4.21 \\
\hline 5. When using this model, all steps of urinary catheter insertion can be carried out. & 0 & 1 & 3 & 5 & 5 & 4.00 \\
\hline 6. I would prefer learning on this simulation model before performing insertion on a real patient. & 0 & 0 & 0 & 4 & 10 & 4.71 \\
\hline 7. I feel more confident in performing catheter insertion. & 0 & 0 & 2 & 8 & 4 & 4.14 \\
\hline 8. I would recommend this simulation to undergraduate medical learners. & 0 & 0 & 0 & 5 & 9 & 4.64 \\
\hline 9. Overall, I found this simulation beneficial. & 0 & 0 & 0 & 8 & 6 & 4.43 \\
\hline
\end{tabular}

model itself and generate a solution for the learning gap identified in the project design. This project was considered a product evaluation and improvement project, and therefore exempt from ethics review.

\section{Results}

Results of the survey are presented in Table 1 .

Surveys were completed by 14 participants who practiced catheter insertion on the 3D-printed model. The average self-reported skill before using the model was 1.29 (out of 5), which increased to 3.21 (out of 5) after using the model (Table 1). Of the 14 participants, $14(100 \%)$ chose either "agree" or "strongly agree" for the following four statements: the simulation was an accurate anatomical representation, they would prefer learning on this simulation model before performing this procedure, they would recommend this model to other learners, and they found this model beneficial overall. In addition, 10 respondents $(71.4 \%)$ would prefer using this model before performing the procedure on an actual patient.

\section{Discussion}

The 3D catheterization model was designed to create a meaningful learning experience as an alternative to existing, commercially available models, with fidelity maximized in the confines of ease and low cost of modifications and replications. Additionally, the 3D printed model incorporates a few unique design features not available in urinary catheter insertion simulators on the market today. The silicone penis is easily manipulated, allowing for straightening of the urethra during insertion of catheter. As the catheter is advanced into the bladder, it passes through a valve that mimics the sphincter muscle, keeping fluid in the bladder without leaking out. Once the sphincter is breached, water will return through the catheter. The end of the catheter can be visualized upon entering the bladder, allowing the user to ensure that the catheter is in the correct position before inflating the balloon. This feature allows the learner to appreciate the drawback of the inflated balloon against the bladder neck, and ensure the catheter is securely in the correct position.

Collectively, these features provide additional sources of visual feedback, which can be beneficial for novice medical learners. ${ }^{14}$ For example, a randomized controlled trial by Zhong et al. compared the use of a transparent urinary tract simulator to a traditional simulator for the teaching of urological skills. ${ }^{15}$ After learning on the transparent simulator, the experimental group scored significantly higher on procedural skills testing than the control group. The ability to visualize the anatomy of the urinary tract and appreciate exactly where the catheter is in relation to important structures is helpful in preventing trauma that results from inflating the balloon while still in the urethra. To that end, one of the major advantages of the 3D simulator is that the learner can see the catheter enter the bladder and inflate the balloon correctly. As a result of the study by Zhong et al and feedback from this iteration of the $3 \mathrm{D}$ catheterization model, a future direction would be incorporating transparent materials in the design of the model.

Using this knowledge, the urinary catheter model we designed will undergo revision and a secondary prototype model will be created. A more rigorous iterative validation process will occur, with a cohort of undergraduate medical and nursing students evaluating the product in tandem with existing high-fidelity models. Undergraduate nursing learners, who will ultimately insert a large majority of catheters in the clinical setting, may also benefit from this model for procedural training. As the simulation model has yet to be formally evaluated by expert users of the procedure (i.e. urologists), its implementation as a learning tool is uncertain; but this early research suggests that a low-fidelity 3D-printed urinary catheter insertion simulation model has the potential to be an effective addition in learning this procedure.

As this iteration of catheter model was intended as a prototype to better inform the identified learning need, the next step in enhancing the simulation experience would be the incorporation of modular elements in the model design. The base of the model would act 
as a static element, allowing for the replacement of individual design pieces such as the urethra or external genitalia. This way, a female model could be designed by swapping out the genitalia and urethra, but keeping the bladder and base, allowing for a quick switch between models. Training for difficult catheterizations would be accomplished by replacing the urethral anatomy to include a stricture or an enlarged prostate, then assessing how a learner would manage this scenario. These modifications can be shared between sites with access to 3D-printing materials, allowing for point-of-care simulation in remote areas without access to more expensive models.

One limitation of this study is the subjective self-assessment of a learner's increased skill in this procedure. This study isn't intended to comment on an objective increase in procedural skill from using this model, but rather as a correlate for increased user confidence, a subjective measure that can result in decreased patient and user anxiety. As many students have never performed a catheter insertion, it is difficult for them to comment on the anatomical accuracy of the model. Also, as one of the future settings for this model may be in nursing education, this study used a cohort of medical students, limiting the applicability of the results obtained.

\section{Conclusion}

This project represents an attempt to ameliorate a learning gap in the preclerkship curriculum of undergraduate medical learners, where a significant portion of respondents indicated that they would feel more comfortable and confident with additional training (including simulated training) in urinary catheter insertion. This procedure can be a stressful experience for both patients and professionals, and increasing user confidence can go a long way towards decreasing patient anxiety. With medical learners being implicated in a significant portion of catheterization related injuries, simulation training offers the advantage of practicing with the ability to make mistakes in a structured setting without the potential for adverse patient events.

\section{References}

1. Canales BK, Weiland D, Reardon S, Monga M. Urethral catheter insertion forces: a comparison of experience and training. Int Braz J Urol. 2009 Feb;35(1):84-9.

2. Feneley RCL, Hopley IB, Wells PNT. Urinary catheters: history, current status, adverse events and research agenda. J Med Eng Technol. 2015 Nov 17;39(8):459-70.

3. Yang RL, Reinke CE, Mittal MK, Kean CR, Diaz E, Fishman NO, et al. The surgery clerkship: an opportunity for preclinical credentialing in urinary catheterization. Am J Surg. 2012 Oct;204(4):535-9.

4. Manalo M, Lapitan MCM, Buckley BS. Medical interns' knowledge and training regarding urethral catheter insertion and insertion-related urethral injury in male patients. BMC Med Educ [Internet]. 2011 Dec [cited 2018 Aug 13];11(1). Available from: http://bmcmededuc.biomedcentral.com/ articles/10.1186/1472-6920-11-73

5. Thomas AZ, Giri SK, Meagher D, Creagh T. Avoidable iatrogenic complications of urethral catheterization and inadequate intern training in a tertiary-care teaching hospital. BJU Int. 2009 Oct 1;104(8):1109-12.

6. Tritschler S, Roosen A, Füllhase C, Stief CG, Rübben H. Urethral Stricture: Etiology, Investigation and Treatments. Dtsch Ärztebl Int. 2013 Mar;110(13):220-6.

7. Rodríguez-Díez MC, Díez N, Merino I, Velis JM, Tienza A, Robles-García JE. Simulators help improve student confidence to acquire skills in urology. Actas Urol Esp Engl Ed. 2014 Jul;38(6):367-72.

8. Schout BMA, Hendrikx AJM, Scheele F, Bemelmans BLH, Scherpbier AJJA. Validation and implementation of surgical simulators: a critical review of present, past, and future. Surg Endosc. 2010 Mar;24(3):536-46.

9. Lichtenberger JP, Tatum PS, Gada S, Wyn M, Ho VB, Liacouras P. Using 3D Printing (Additive Manufacturing) to Produce Low-Cost Simulation Models for Medical Training. Mil Med. 2018 Mar 1;183(suppl_1):73-7.

10. Grober ED, Hamstra SJ, Wanzel KR, Reznick RK, Matsumoto ED, Sidhu RS, et al. The Educational Impact of Bench Model Fidelity on the Acquisition of Technical Skill: The Use of Clinically Relevant Outcome Measures. Ann Surg. 2004 Aug;240(2):374-81.

11. Badash I, Burtt K, Solorzano CA, Carey JN. Innovations in surgery simulation: a review of past, current and future techniques. Ann Transl Med [Internet]. 2016 Dec 16 [cited 2018 Jun 11];4(23). Available from: http://atm.amegroups.com/ article/view/12868

12. Doucet G, Ryan S, Bartellas M, Parsons M, Dubrowski A, Renouf T. Modelling and Manufacturing of a 3D Printed Trachea for Cricothyroidotomy Simulation. 2017;14.

13. Berg K, Berg D, Riesenberg LA, Mealey K, Schaeffer A, Weber D, et al. The Development of Validated Checklist for Foley Catheterization: Preliminary Results. Am J Med Qual. 2013;28(6):519-524.

14. Guadagnoli M, Morin M-P, Dubrowski A. The application of the challenge point framework in medical education. Med Educ 2012 May 1;46(5):447-53.

15. Zhong X, Wang P, Feng J, Hu W, Huang C. Novel Transparent Urinary Tract Simulator Improves Teaching of Urological Operation Skills at a Single Institution. Urol Int. 2015 Feb 26;95(1):38-43. 